\title{
Saliency detection of infrared image based on region covariance and global feature
}

\author{
LIU Songtao*, JIANG Ning, LIU Zhenxing, and JIANG Kanghui
}

Department of Information Operation, Dalian Naval Academy, Dalian 116018, China

\begin{abstract}
In order to better represent infrared target features under different environments, a saliency detection method based on region covariance and global feature is proposed. Firstly, the region covariance features on different scale spaces and different image regions are extracted and transformed into sigma features, then combined with central position feature, the local salient map is generated. Next, a global salient map is generated by gray contrast and density estimation. Finally, the saliency detection result of infrared images is obtained by fusing the local and global salient maps. The experimental results show that the salient map of the proposed method has complete target features and obvious edges, and the proposed method is better than the state of art method both qualitatively and quantitatively.
\end{abstract}

Keywords: saliency detection, region covariance, gray contrast, density estimation.

DOI: $10.21629 / J S E E .2018 .03 .05$

\section{Introduction}

Most of the infrared image targets are salient, but due to the imaging conditions and the impact of the surrounding environment, some of the infrared target areas has obvious gray differences, blurred edges, or its surrounding background is more salient. Therefore, it is a noteworthy research direction for making target detection and segmentation simple through highlighting the characteristics of infrared targets.

The saliency of the image area includes uniqueness, scarcity and singularity, which has attracted the attention of many scholars. The model of saliency computation mainly includes bottom-up methods and top-down methods. The most classical method of the bottom-up model is the Itti model [1]. The center-surround operator and the Gaussian pyramid are used to make the colors, intensity and

Manuscript received March 02, 2017.

*Corresponding author.

This work was supported by the National Natural Science Foundation of China (61303192) and the China Postdoctoral Science Foundation (2015M572694; 2016T90979). orientations of each feature channel firstly, then based on the feature integration theory, the conspicuity maps are obtained and fused for the final saliency map. Since then, many saliency computational models based on local contrast are put forward, for example, Ma et al. [2] proposed the use of the difference between the current pixel and the surrounding neighborhood pixels to calculate the pixels' saliency. Since the local contrast method ignores the global feature, such methods usually have obvious edges, rather than uniformly highlighting the entire target. Thus, the saliency methods by calculating the global contrast appear. Hou et al. [3] proposed a saliency map generation algorithm based on Fourier transform, which mainly analyzes the amplitude spectrum, and Guo et al. [4] developed the residual spectral method, using only the phase information of Fourier transform. However, these methods ignore the spatial coherence of the target. Thus, Jung et al. [5] proposed a joint frequency domain method, which uses the local spectral features to extract structural information. With regard to spatial coherence, it can also be considered from the spatial domain. The salient target should be compact in space, and the background color should be distributed throughout the image. The methods for calculating spatial coherence include the variance of spatial position [6], twodimensional entropy [7], similarity distribution [8] and local density [9]. In view of the different advantages of local saliency and global saliency, Li et al. [10] proposed a saliency model by combination of global features and local features, which uses frequency-domain smoothing to suppress the non-salient area, and the local filter based on independent component analysis (ICA) to enhance the target area. The results of the above salient maps are either salient target's edges or obvious target's center, and the entire target is not salient. However, the region-based saliency computational models can solve this problem. Cheng et al. [11] defined the region saliency by the contrast and spatial relationship of the current region and other regions, and these 
regions are obtained by the super-pixel segmentation in advance. Similarly, Perazzi et al. [12] proposed a saliency filter method, which obtained the contrast features using super-pixel coherence and spatial distribution.

The top-down saliency computational methods mainly view saliency detection as a learning problem. Liu et al. [13] used the conditional random field (CRF) framework to study the weights of local features, global features, and regional features, and then adopt the trained CRF to detect the saliency of image targets. Jiang et al. [14] considered the salient target detection as a regression problem, and a regression is used to map the regional feature vector directly to a salient value. In addition, the top-down information can be modeled, Zhang et al. [15] proposed to use the Bayesian framework to detect salient targets, where the information of the known target is modeled as a likelihood function.

In [16], a saliency detection method for different scale spaces is constructed with the region covariance feature, which reflects the local saliency of the image object better. When this method is applied to the detection of infrared image saliency, it is found that the target area is inconsistently salient and the edges are blurred. In order to overcome these shortcomings, this paper combines the mean of the feature vectors and the sigma features to generate the local saliency map. At the same time, the global saliency map based on gray contrast and density estimation is introduced, and finally the local and global saliency maps are fused to realize the saliency detection. The experimental results show that the novel method is an effective method for saliency detection of infrared images.

\section{Local saliency detection based on region covariance}

Because the region covariance can naturally fuse a number of related features, and the covariance calculation itself has the filtering ability and high efficiency [17], this paper uses the region covariance feature to implement local saliency detection.

\subsection{Region covariance feature}

The infrared image is divided by a rectangular grid, then the following features are extracted for each pixel in each rectangular region, such as the gray of the image, the norm of the first and second order gradients in the $x$ and $y$ directions, so each pixel is mapped to the $d=5$ dimension feature vector [18] as follows:

$$
\begin{gathered}
\boldsymbol{F}(x, y)=\left[\boldsymbol{I}(x, y)\left|\frac{\partial \boldsymbol{I}(x, y)}{\partial x}\right|\left|\frac{\partial \boldsymbol{I}(x, y)}{\partial y}\right| .\right. \\
\left.\left|\frac{\partial^{2} \boldsymbol{I}(x, y)}{\partial x^{2}}\right|\left|\frac{\partial^{2} \boldsymbol{I}(x, y)}{\partial y^{2}}\right|\right]^{\mathrm{T}}
\end{gathered}
$$

where $\boldsymbol{I}$ is the image gray scale, and the image gradient is calculated by the filter $\left[\begin{array}{lll}-1 & 0 & 1\end{array}\right]^{\mathrm{T}}$ and $\left[\begin{array}{lll}-1 & 2 & -1\end{array}\right]^{\mathrm{T}}$.

According to the feature vectors in each rectangular region, its covariance matrix is calculated as follows:

$$
\boldsymbol{C o v}_{R}=\frac{1}{n-1} \sum_{p=1}^{n}\left(\boldsymbol{f}_{p}-\boldsymbol{\mu}\right)^{\mathrm{T}}\left(\boldsymbol{f}_{p}-\boldsymbol{\mu}\right)
$$

where $\boldsymbol{\mu}$ is the mean of the feature vectors and $n$ is the number of region pixels.

In order to make the region covariance reflect the image region characteristics better and facilitate the similarity calculation, Hong et al. [19] used Cholesky decomposition to convert the covariance matrix to the Euclidean space, designed the sigma feature, and introduced the first-order statistical mean to obtain the enhanced sigma feature. Let $C$ be the covariance matrix with dimension $d \times d$, and the sigma feature is calculated as

$$
s_{i}=\left\{\begin{array}{l}
\alpha \sqrt{d} \boldsymbol{L}_{i}, \quad 1 \leqslant i \leqslant d \\
-\alpha \sqrt{d} \boldsymbol{L}_{i}, \quad d+1 \leqslant i \leqslant 2 d
\end{array}\right.
$$

where $\boldsymbol{L}_{i}$ is the $i$ th column of the matrix $\boldsymbol{L}, \boldsymbol{C}=\boldsymbol{L} \boldsymbol{L}^{\mathrm{T}}$, $\alpha$ is the coefficient. When the mean of the $d$-dimensional eigenvector is introduced, the enhanced sigma eigenvector is as follows:

$$
\begin{gathered}
\psi(\boldsymbol{C})= \\
\left(\boldsymbol{s}_{1}+\boldsymbol{\mu}, \ldots, \boldsymbol{s}_{d}+\boldsymbol{\mu}, \boldsymbol{s}_{d+1}+\boldsymbol{\mu}, \ldots, \boldsymbol{s}_{2 d}+\boldsymbol{\mu}\right)^{\mathrm{T}} .
\end{gathered}
$$

\subsection{Local saliency detection}

The saliency of the region $\boldsymbol{R}_{i}$ is defined as the weighted average of the dissimilarity degree of the region and its nearest $m$ neighborhood [16]. The specific formula is as follows:

$$
\boldsymbol{S}_{l}\left(\boldsymbol{R}_{i}\right)=\frac{1}{m} \sum_{j=1}^{m} \boldsymbol{L}\left(\boldsymbol{R}_{i}, \boldsymbol{R}_{j}\right)
$$

where the $m$ most similar regions of the region $\boldsymbol{R}_{i}$ can be determined according to the dissimilarity measure $\boldsymbol{L}\left(\boldsymbol{R}_{i}, \boldsymbol{R}_{j}\right)$, whose expression is as follows:

$$
\boldsymbol{L}\left(\boldsymbol{R}_{i}, \boldsymbol{R}_{j}\right)=\frac{\left\|\boldsymbol{\psi}\left(\boldsymbol{C}_{i}\right)-\boldsymbol{\psi}\left(\boldsymbol{C}_{j}\right)\right\|}{1+\left\|\boldsymbol{x}_{i}-\boldsymbol{x}_{j}\right\|}
$$

where $C_{i}$ and $C_{j}$ are the covariance matrices of the region $\boldsymbol{R}_{i}$ and $\boldsymbol{R}_{j}, \boldsymbol{x}_{i}$ and $\boldsymbol{x}_{j}$ are the central coordinates of these two regions. In (6), the sigma feature distance weighted by the distances between these regions considers that the spatial distance has effects on the inter-region similarity.

\section{Global saliency detection based on gray contrast and density estimation}

The gray contrast feature can better reflect the global saliency of the image. Therefore, this paper uses the gray contrast feature and density estimation to generate a global saliency map with spatial coherence [9]. 


\subsection{Gray contrast feature}

The saliency of a pixel is defined using its color contrast to all other pixels in the image. Specially, the pixel saliency (PS) of $\boldsymbol{I}_{l}$ in image $\boldsymbol{I}$ is defined as

$$
\boldsymbol{P} \boldsymbol{S}\left(\boldsymbol{I}_{l}\right)=\sum_{\forall \boldsymbol{I}_{i} \in \boldsymbol{I}} \boldsymbol{D}\left(\boldsymbol{I}_{l}, \boldsymbol{I}_{i}\right)
$$

where $\boldsymbol{D}\left(\boldsymbol{I}_{l}, \boldsymbol{I}_{i}\right)$ is the color distance metric between pixels $\boldsymbol{I}_{l}$ and $\boldsymbol{I}_{i}$. It is easy to see that the pixels with the same gray-level have the same saliency values. Thus we rearrange (7) such that terms with the same gray-level $c_{j}$ are grouped together, we get the saliency value for each gray-level as

$$
\boldsymbol{P} \boldsymbol{S}(\boldsymbol{I}(x, y))=\boldsymbol{P} \boldsymbol{S}\left(c_{k}\right)=\sum_{j=1}^{u} f_{j} \boldsymbol{D}\left(c_{k}, c_{j}\right)
$$

where $c_{k}$ is the gray value of pixel $\boldsymbol{I}(x, y), u$ is the number of different gray-level, and $f_{j}$ is the probability of graylevel $c_{j}$ in image $\boldsymbol{I}$.

\subsection{Density estimation}

It has been observed that the region with high density is more salient than that of low density in the same gray-level. Thus the local density is proposed to describe the spatial coherence. When the local density is large, it means that the pixels among this local region are more salient. The specific formula is as follows:

$$
\boldsymbol{S C}\left(c_{k}\right)=\frac{\max \boldsymbol{N}\left(r_{k, v}\right)}{\sum_{k=1}^{u} \max \boldsymbol{N}\left(r_{k, v}\right)}
$$

where $r_{k, v}$ is the $v$ th region in the $k$ th gray-level, $\boldsymbol{N}\left(r_{k, v}\right)$ is the pixels' number of region $r_{k, v}$, which is viewed as the local density.

\subsection{Global saliency detection}

Equation (8) only describes the PS by the histogram based contrast feature, while the spatial distribution characteristics are also important effect to the salient object, which can be described by spatial coherence using (9). Therefore we obtain the global saliency map by combining PS and spatial coherence as follows:

$$
\boldsymbol{S}_{g}(\boldsymbol{I}(x, y))=\boldsymbol{S}\left(c_{k}\right)=\boldsymbol{S C}\left(c_{k}\right) * \sum_{j=1}^{u} f_{j} \boldsymbol{D}\left(c_{k}, c_{j}\right)
$$

\section{Saliency detection by fusing local and global features}

In order to highlight the infrared target characteristics under complex environments better, this paper will expand the local saliency based on regional covariance, and combine with the global saliency to achieve the final saliency detection.

(i) Saliency weighted by central position. The pixels near the center of the image are usually more pronounced than the pixels on the edges, so the central position feature is introduced to modify the local saliency [16], the formula is

$$
\boldsymbol{S}_{l}^{\prime}\left(\boldsymbol{R}_{i}\right)=\left(1-\frac{\left\|\boldsymbol{x}_{i}-\boldsymbol{x}_{c}\right\|}{Z}\right) \cdot \boldsymbol{S}_{l}\left(\boldsymbol{R}_{i}\right)
$$

where $\boldsymbol{x}_{c}$ is the coordinates of the image center, and $Z=$ $\max _{i^{\prime} \in I}\left\|\boldsymbol{x}_{i^{\prime}}-\boldsymbol{x}_{c}\right\|$ is the normalized coefficient.

(ii) Fusion with scale space saliency. Different sizes of grids can extract salient targets at different scales, and the target's size in the infrared image is unknown. Therefore, it is considered that the local saliency map should be generated by different scale spaces. Furthermore, since the salient target should be salient at all scales, the local saliency map is obtained by

$$
\boldsymbol{S}_{l}(x, y)=\prod_{k \in K} \boldsymbol{S}_{l}^{k}(x, y)
$$

where $k$ is the parameter of the scale space, $K$ is the set of the scale space, the size of the image region $W=H=$ $\frac{256}{k}, \boldsymbol{S}_{l}^{k}(x, y)$ is the pixel salient value at the scale space $k$. Here, the size of the input image should be resized to $256 \times 256$.

(iii) Fusion with local and global saliency maps. By introducing the mean of the feature vector into the sigma feature, the saliency of the target area can be consistent, but the background clutter is obvious. In order to suppress the background clutter better and adapt to the target's size, the central position feature and scale space mechanism are introduced. In addition, the local saliency cannot embody the salient extent of the target in the whole image, which results in litter clutter residues. Considering that the interested target is usually rare in the infrared image, so it is necessary to introduce the global saliency for suppressing the background clutter, and making the edge of the target area obvious. According to the above ideas, the combination of local and global saliency maps is as follows:

$$
\boldsymbol{S}(x, y)=\boldsymbol{S}_{l}(x, y) \cdot \boldsymbol{S}_{g}(\boldsymbol{I}(x, y))
$$

The flow chart of the whole saliency detection algorithm is shown in Fig. 1. The main features of the new method are as follows: (i) local saliency detection is realized by the enhanced sigma feature; (ii) global saliency detection is based on gray contrast and density estimation; (iii) the final saliency map is obtained by fusing local and global saliency maps, resulting in that the saliency of the target 
region is consistent and the edges are obvious. In short, the proposed method can perform saliency detection more effectively.

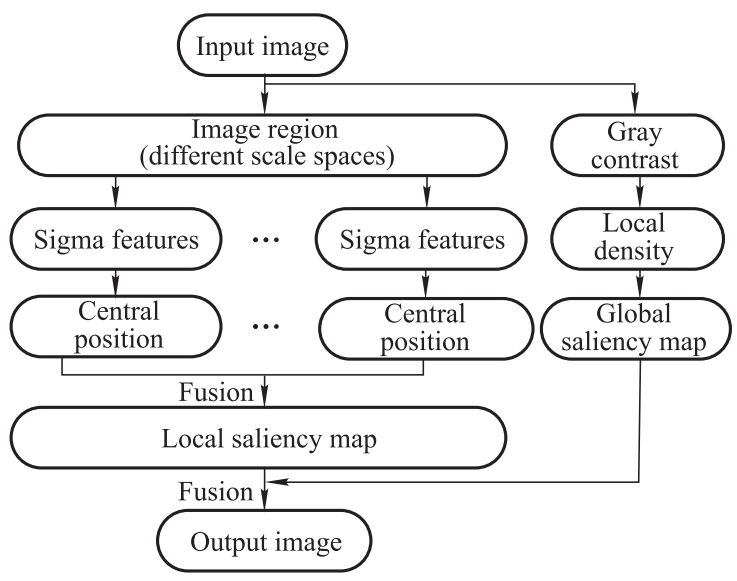

Fig. 1 Flow chart of saliency detection algorithm

\section{Experimental results}

Firstly, the analysis of the saliency map is used to illustrate the improvement of the novel method on the method in [16], and then the advantages of the proposed method are compared with that of state-of-the-art methods both qualitatively and quantitatively. The experimental images are collected by a long-wave infrared camera, whose type is JIR-1161, with focal length $100 \mathrm{~mm}$, band range $8-14$ um, viewing angle $9.15^{\circ} \times 6.87^{\circ}$, pixel number $640 \times$ 480 , and shooting objects includes icebreaker, ordinary freighter, small fishing boat and plane. The novel algorithm's parameters are mainly $m, K$ and $\alpha$. In the experiment, $m=0.1 \times N u m, N u m$ is the number of all neighborhood regions of the current region, $K=\{64,32,16\}$, $\alpha=\sqrt{2}$.

\subsection{Performance analysis of the proposed algorithm}

Fig. 2(a) is an infrared image of a plane target with a cloudy background. The saliency detection results of the method in [16] are shown in Fig. 2(b). It is obvious that the saliency of the target area is inconsistent and the background clutter is obvious. After introducing the mean of the feature vector into the sigma features, the new saliency map is shown in Fig. 2(c), where the saliency of the target area is consistent, but the background clutter is more obvious. In order to suppress the background clutter, and make the target's edge obvious, the global feature is introduced. The saliency result of the global feature is shown in Fig. 2(d), where the saliency of the target area is consistent and the edge is clear, but there is a lot of background clutter. The proposed method of this paper combines the global saliency map and the local saliency map. The result is shown in Fig. 2(e), where the saliency of the target area is consistent, the edge is obvious, and the background clutter is also suppressed. Therefore, this novel method is effective for the improvement of the method in [16].

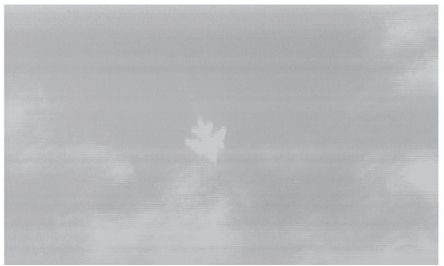

(a) Original image

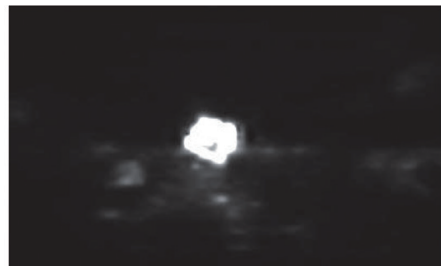

(b) The method in [16]

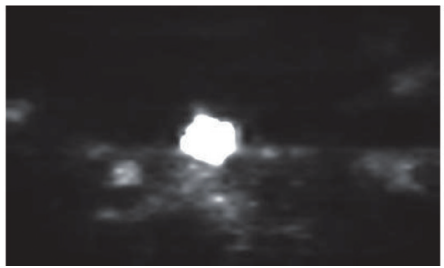

(c) Enhanced sigma feature

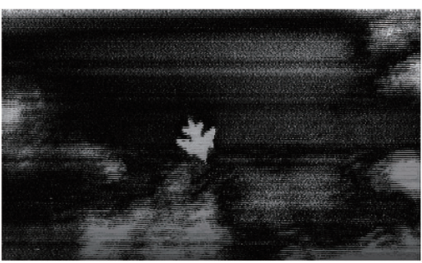

(d) Global feature

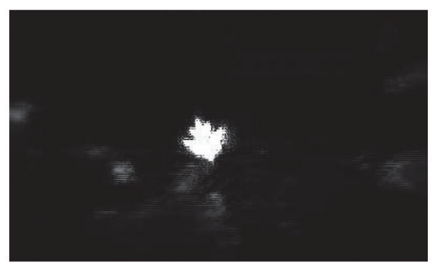

(e) The propsed method

Fig. 2 Saliency maps of four saliency detection methods

However, regardless of the proposed method, or the method in [16], the computational time is somewhat long and about $10 \mathrm{~s}$ due to the multi-scale mechanism. At the time of simulation, the hardware performance of the laptop is $2.3 \mathrm{GHz}$ CPU, $8 \mathrm{G}$ memory, and its operating system is win732-bit and the program is developed by Matlab 7.6. Because the target size of the infrared image is unknown, multi-scale is necessary, see Fig. 3. For small targets, a large scale will make the target's saliency disappear, see Fig. 3(b). For large targets, a small scale will make the edges blurred, and the clutter is obvious, see Fig. 3(c). Regardless of the target size, multi-scale can be better to detect the target's saliency, see Fig. 3(d). 

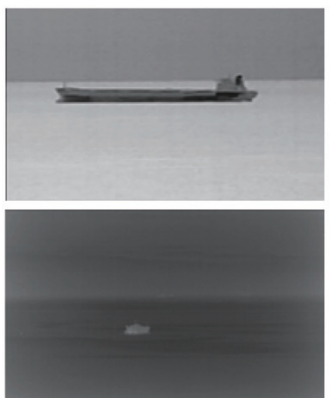

(a) Original image
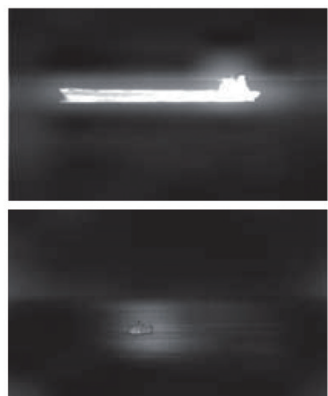

(b) Big scale

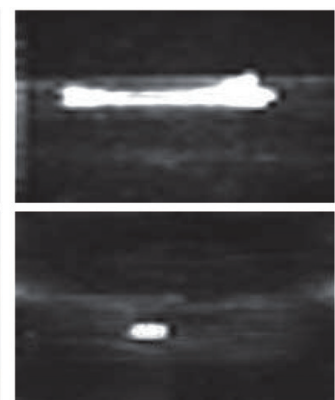

(c) Small scale

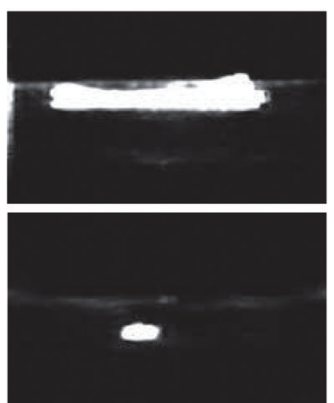

(d) Multiple scales

Fig. 3 Saliency maps of different scale spaces

For the saliency detection results in Fig. 2 and Fig. 3, the weighted F-measure metric [20] is adopted for objective evaluation, which is a weighted version of the traditional F-measure. The larger the value of the F-measure, the bet- ter the saliency detection results. The evaluation results are shown in Table 1. It is obvious that the objective and subjective results are consistent, and the proposed method can get better results.

Table 1 Weighted F-measure of the saliency detection results

\begin{tabular}{cccccccccc}
\hline Evaluation index & Fig. 2(b) & Fig. 2(c) & Fig. 2(d) & Fig. 2(e) & Fig. 3(b) & Fig. 3(c) & Fig. 3(d) & Row \\
\hline \multirow{2}{*}{ F-measure } & \multirow{2}{*}{0.3369} & \multirow{2}{*}{0.1856} & \multirow{2}{*}{0.0372} & \multirow{2}{*}{0.5899} & 0.4029 & 0.4068 & 0.5009 & The first row \\
\cline { 5 - 9 } & & & & & & & & & \\
\end{tabular}

\subsection{Comparison to state-of-the-art saliency detection methods}

In order to evaluate the superiority of this proposed method more widely, the saliency detection results for different infrared images are analyzed using the twelve saliency detection methods, i.e., frequency [21], graph-based visual saliency (GBVS) [22], spatial coherence [9], hypercomplex Fourier transform (HFT) [23], itti [1], the maximum symmetric surround (MSS) [24 ], saliency measure [25], signature [26], saliency by induction mechanisms (SIM) [27], spectral [3], covarince [16], covariance global (the proposed method). Fig. 4 is the saliency map of the twelve methods.

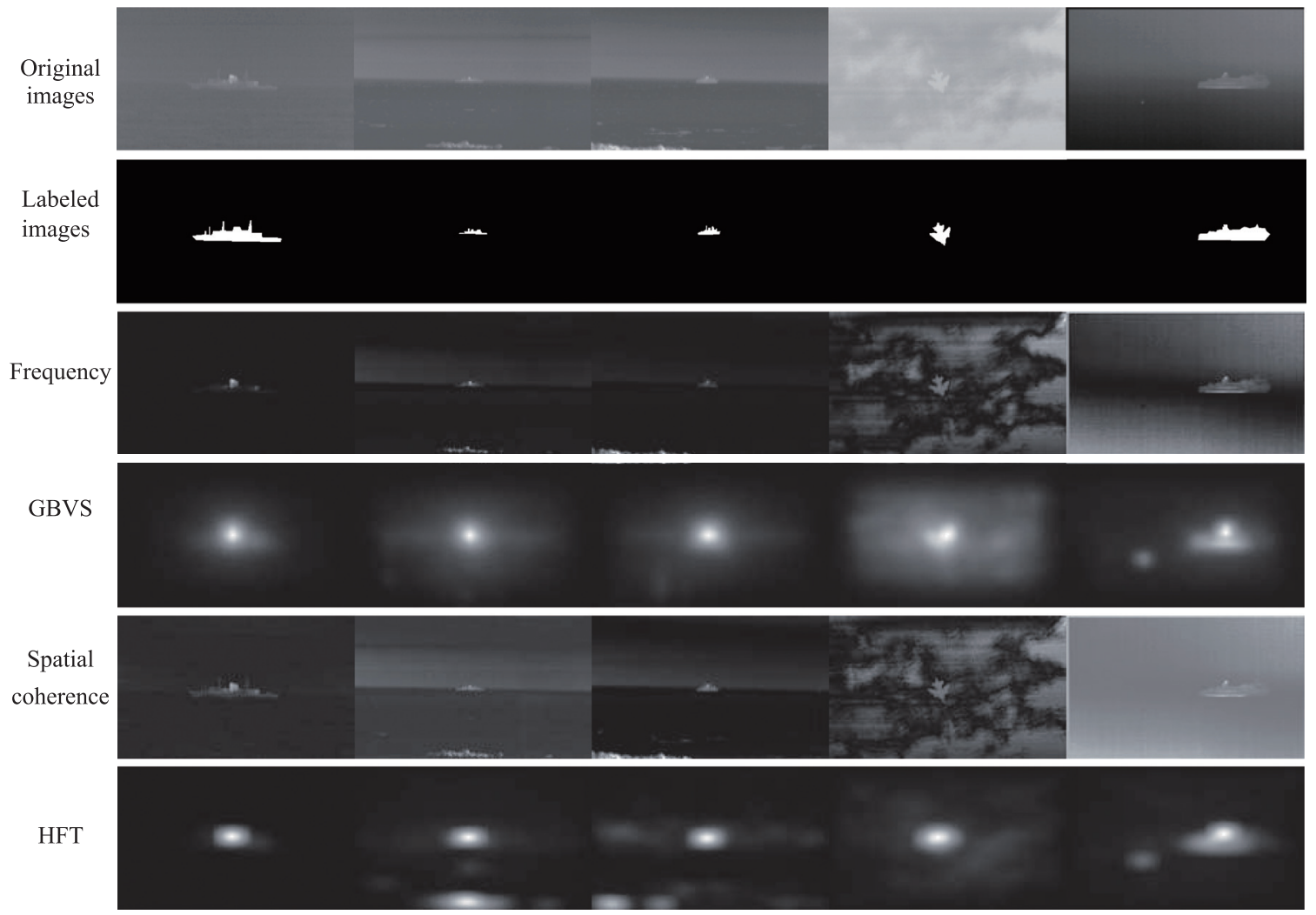




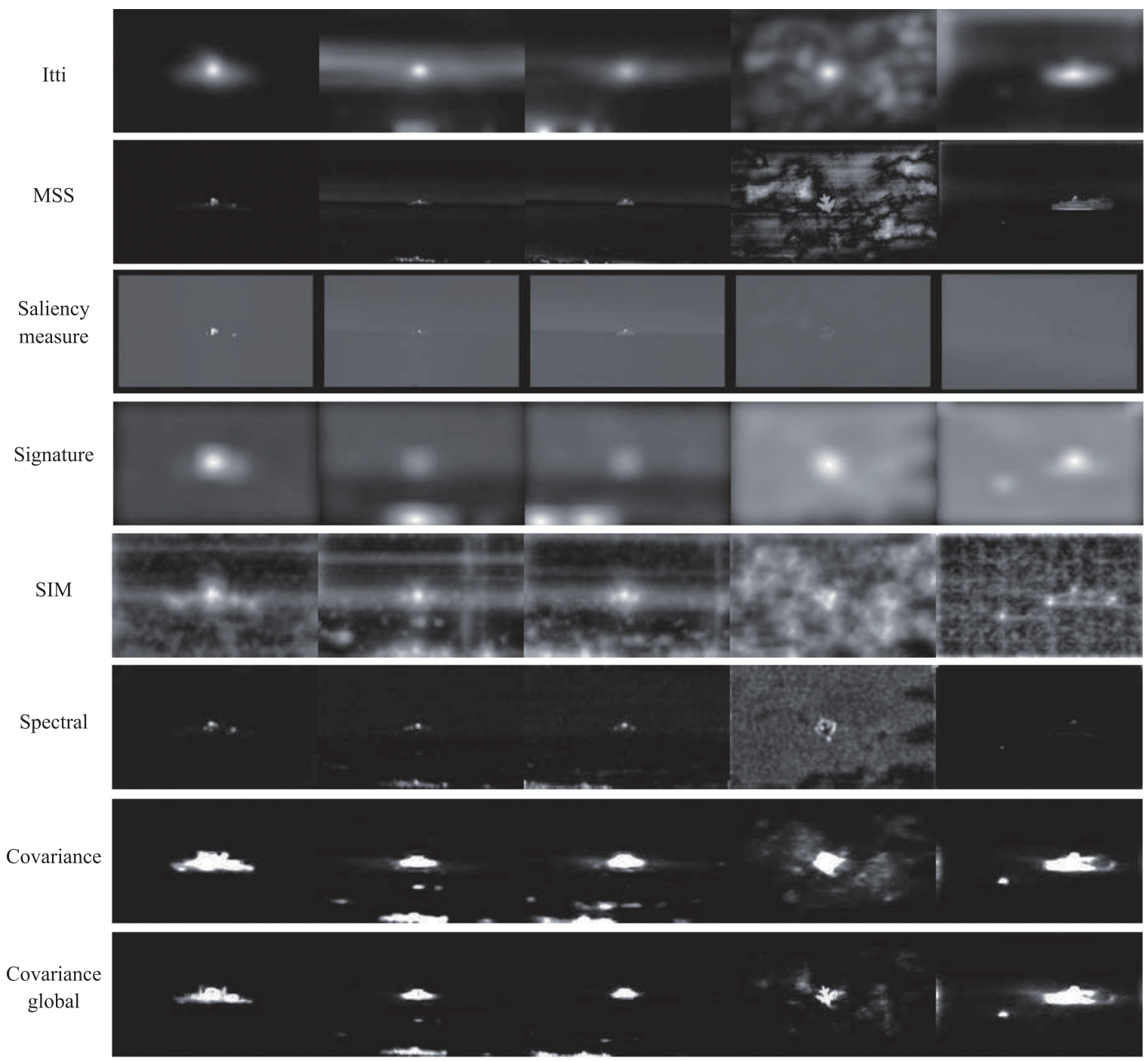

Fig. 4 Saliency maps of twelve saliency detection methods

The results of these methods (frequency, MSS, and spectral) have bright points and edges, in which the MSS method has the best effect; the results of these methods (GBVS, HFT, itti, signature, and SIM) make the target area salient, in which the results of these methods (SIM and signature) are serious in background clutter, and the result of the itti's method has good target shape, but its background clutter is relatively large, and the result of these methods (HFT and GBVS) have the most accurate target information, but the HFT's method has no target shape information, and the GBVS's method has obvious clutter; the results of these methods (spatial coherence and saliency measure) have obvious target details but the whole target is not salient; the result of the covariance's method is similar to that of the proposed method, where the target area is well and the target shape is complete. However, the covari- ance's method has serious clutter and blurred edges, and the proposed method can avoid these deficiency. Hence, the proposed method is optimal in these twelve saliency detection methods.

In order to further evaluate the superiority of the proposed method, the Precision and Recall (PR) curve is used to compare the effects of different methods, and Fig. 5 is the PR curve of these 12 methods. The higher the starting position of the PR curve and the larger the area below the envelope, the better the performance of the saliency detection algorithm. Obviously, the covariance's method and the proposed method are optimal, especially the proposed method has optimal precision for different recalls, which further validates the subjective evaluation of the saliency map, i.e., the proposed method has the best infrared image saliency detection performance. 


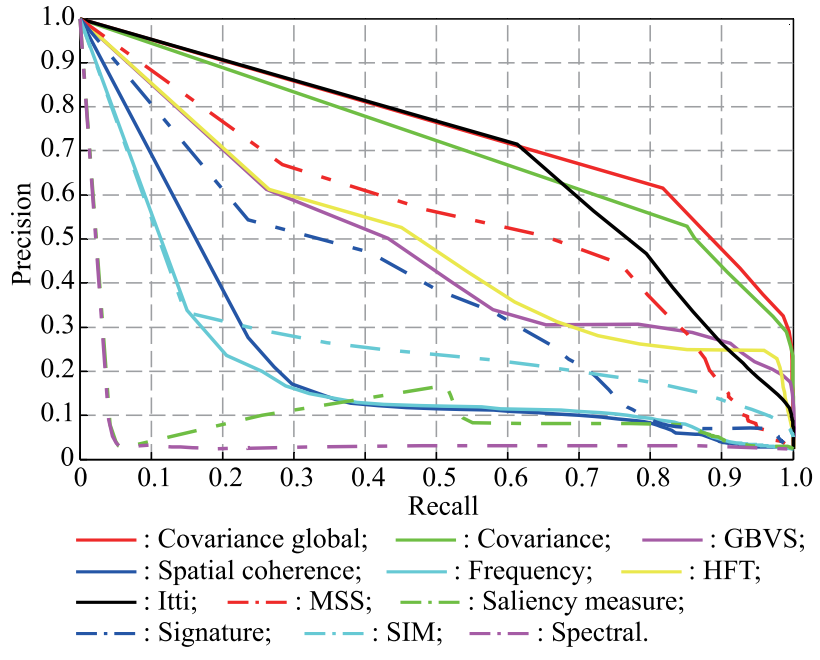

Fig. 5 PR curves of twelve saliency detection methods

\section{Conclusions}

In this paper, a saliency detection method based on region covariance is used to enhance the target characteristics of infrared images, and an infrared image saliency detection method is proposed by fusing the region covariance and global features. On the basis of the saliency detection method of region covariance, the proposed method combines the mean of the feature vector and the sigma feature to generate the local salient map, and introduces the global salient map based on the gray contrast and density estimation, and finally integrates the local and global saliency maps to realize the saliency detection. The experimental results show that the results of the proposed method have consistent target information and obvious edges. The new method is superior to state-of-the-art saliency detection methods both qualitatively and quantitatively, and its main problem is time-consuming, so the future work may consider the use of other methods, such as super-pixel preprocessing or scale space reduction [28], to reduce the amount of calculation.

\section{References}

[1] ITTI L, KOCH C, NIEBUR E. A model of saliency based visual attention for rapid scene analysis. IEEE Trans. on Pattern Analysis and Machine Intelligence, 1998, 20 (11): 1254 1259.

[2] MA Y, ZHANG H. Contrast-based image attention analysis by using fuzzy growing. Proc. of the 11th ACM International Conference on Multimedia, 2003: 374-381.

[3] HOU X, ZHANG L. Saliency detection: a spectral residual approach. Proc. of the IEEE Conference on Computer Vision and Pattern Recognition, 2007: 1-8.

[4] GUO C L, MA Q, ZHANG L M. Spatio-temporal saliency detection using phase spectrum of quaternion fourier transform. Proc. of the IEEE Conference on Computer Vision and Pattern Recognition, 2008: 1-8.
[5] JUNG C H, KIM C I. A unified spectral-domain approach for saliency detection and its application to automatic object segmentation. IEEE Trans. on Image Processing, 2012, 21(3): $1272-1283$.

[6] HE X, JING H Y, HAN Q, et al. Salient region detection combining spatial distribution and global contrast. Optical Engineering, 2012, 51(4): 047007.

[7] ZHANG Y B, HAN J W, GUO L. Image saliency detection based on histogram. Journal of Computational Information Systems, 2014, 10(6): 2417-2424.

[8] XUA L F, LIA H L, ZENG L Y, et al. Saliency detection using joint spatial-color constraint and multi-scale segmentation. Journal of Visual Communication and Image Representation, 2013, 24(4): $465-476$.

[9] LIU S T, SHEN T S, DAI Y. Infrared image segmentation method based on spatial coherence histogram and maximum entropy. Proc. of the Photonics Asia Conference, 2014: 1-8.

[10] LI J, LEVINE M D, AN X J, et al. Saliency detection based on frequency and spatial domain analysis. Proc. of the British Machine Vision Conference, 2011: 1-11.

[11] CHENG M M, ZHANG G X, MITRA N J, et al. Global contrast based salient region detection. Proc. of the International Conference on Computer Vision, 2011: 409-416.

[12] PERAZZI F, KRAHENBUHL P, PRITCH Y, et al. Saliency filters: contrast based filtering for salient region detection. Proc. of the IEEE Conference on Computer Vision and Pattern Recognition, 2012: $733-740$.

[13] LIU T, SUN J, ZHENG N, et al. Learning to detect a salient object. Proc. of the IEEE Conference on Computer Vision and Pattern Recognition, 2007: 1-8.

[14] JIANG H, WANG J, YUAN Z, et al. Salient object detection: a discriminative regional feature integration approach. Proc. of the IEEE Conference on Computer Vision and Pattern Recognition, 2013: 2083 - 2090.

[15] ZHANG L, TONG M, MARKS T, et al. SUN: a bayesian framework for saliency using natural statistics. Journal of Vision, 2008, 8(7): 1-20.

[16] ERDEM E, ERDEM A. Visual saliency estimation by nonlinearly integrating features using region covariances. Journal of Vision, 2013, 13(4): 1-20.

[17] TUZEL O, PORIKLI F, MEER P. Region covariance: a fast descriptor for detection and classification. Proc. of the European Conference on Computer Vision, 2006: 589-600.

[18] LIU S T, CHANG C, SHEN T S. The image feature fusion method based on region covariance. Electronics Optics \& Control, 2015, 22(2): 7-11. (in Chinese)

[19] HONG X, CHANG H, SHAN S, et al. Sigma set: a small second order statistical region descriptor. Proc. of the IEEE Conference on ComputerVision and Pattern Recognition, 2009: $1802-1809$.

[20] MARGOLIN R, ZELNIK-MANOR L, TAL A. How to evaluate foreground maps. Proc. of the IEEE Conference on Computer Vision and Pattern Recognition, 2014: 248-255.

[21] ACHANTA R, HEMAMI S, ESTRADA F, et al. Frequencytuned salient region detection. Proc. of the IEEE Conference on Computer Vision and Pattern Recognition, 2009: 1597 1604.

[22] SCHOLKOPF B, PLATT J, HOFMANN T. Graph-based visual saliency. Advances in Neural Information Processing Systems, 2006, 19: $545-552$.

[23] LI J, LEVINE M D, AN X J, et al. Visual saliency based on scale-space analysis in the frequency domain. IEEE Trans. on Pattern Analysis \& Machine Intelligence, 2013, 35(4): $996-$ 1010.

[24] ACHANTA R, SUSSTRUNK S. Saliency detection using 
maximum symmetric surround. Proc. of the IEEE International Conference on Image Processing, 2010: 2653-2656.

[25] RAHTU E, KANNALA J, SALO M, et al. Segmenting salient objects from images and videos. Proc. of the European Conference on Computer Vision, 2010: 366-379.

[26] HOU X, HAREL J, KOCH C. Image signature: highlighting sparse salient regions. IEEE Trans. on Pattern Analysis \& Machine Intelligence, 2012, 34(1): 194-201.

[27] MURRAY N, VANRELL M, OTAZU X, et al. Saliency estimation using a non-parametric low-level vision model. Proc. of the IEEE Conference on Computer Vision and Pattern Recognition, 2011: $433-440$.

[28] JING H, HE X, HAN Q, et al. Saliency detection based on integrated features. Neurocomputing, 2014, 129(4): 114-121.

\section{Biographies}

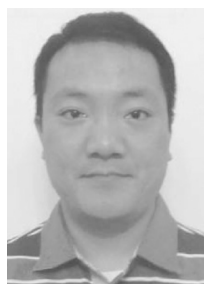

LIU Songtao was born in 1978. He received his B.S. degree in aviation radar, M.S. degree and Ph.D. degree in signal and information processing from Naval Aeronautical Engineering Institute, in 2000, 2003 and 2006, respectively. He joined the Dalian Naval Academy in 2006 where he is now an associate professor in the Department of Information Operation. From 2010 to 2012, he was a postdoctoral researcher at the Postdoctoral Station of Information and Communication Engineering, Dalian University of Technology. He has published over 70 papers on journals and conference proceedings. His research interests include image processing, optoelectronic engineering, and electronic countermeasures.

E-mail: navylst@163.com

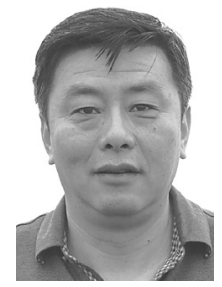

JIANG Ning was born in 1964. He has been with the Dalian Naval Academy since 1989. He received his B.S. degree in electronic countermeasure engineering from Naval Electronic Engineering Academy in 1987, and M.S. degree and Ph.D. degree in computer application and system engineering from Dalian University of Technology in 1996 and 2000, respectively. From 2000 to 2002, he was a postdoctoral researcher at the Postdoctoral Station of Control Science and Engineering, Nanjing University of Technology. He became an associate professor in 2002 and has been a professor since 2007. His research interests include electronic countermeasure and information operation. E-mail: jiangning68@sohu.com

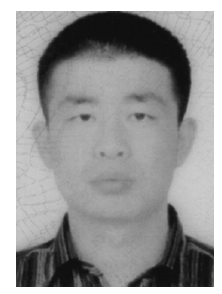

LIU Zhenxing was born in 1980. He received his B.S., M.S. and Ph.D. degrees in operation command from Dalian Naval Academy (DNA) in 2002, 2007 and 2010, respectively. Since 2010, he has been a lecturer at the Department of Information Operation, DNA. His research interests include optoelectronic engineering and electronic countermeasures.

E-mail: liuzhenxing@msn.com

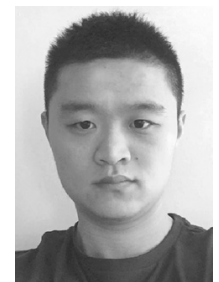

JIANG Kanghui was born in 1991. He received his B.S. degree in information engineering from Hohai University in 2015. Now he is a postgraduate in information and communication engineering from Dalian Naval Academy. His research interest includes image processing.

E-mail: 1284268886@qq.com 